\title{
THE DESCENDING CHAIN CONDITION \\ IN MODULAR LATTICES
}

THOMAS G. NEWMAN

(Received 5 May 1970)

Communicated by $\mathbf{B}$. Mond

In a recent paper Kovács [1] studied join-continuous modular lattices which satisfy the following conditions:

(i) every element is a join of finitely many join-irredicibles, and,

(ii) the set of join-irreducibles satisfies the descending chain condition. He was able to prove that such a lattice must itself satisfy the descending chain condition. Interest was expressed in whether or not one could obtain the same result without the assumption of modularity and/or of join-continuity. In this paper we give an elementary proof of this result without the assumption of joincontinuity (which of course must then follow as a consequence of the descending chain condition). In addition we give a suitable example to show that modularity may not be omitted in general. We first state the main result:

THEOREM. If Lis a modular lattice in which (i) and (ii) hold, then L satisfies the descending chain condition.

The proof will be given after establishing a preliminary result.

Lemma. Let $L$ be a modular lattice and let $K$ be the set of all $x \in L$ such that the principal ideal $(x)$ generated by $x$ satisfies the descending chain condition. Then $K$ is an ideal of $L$ (possibly void). Moreover,Ksatisfies the descending chain condition.

PROOF. It is enough to prove that $K$ is join closed since everything else is obvious and holds in general. Let $a, b \in K$ and suppose we are given a chain $a \vee b \geqq x_{1} \geqq x_{2} \geqq \cdots$. Observe that the descending chain condition holds in the interval $[a, a \vee b]$ - by the isomorphism theorem in modular lattices-since it is transposed to $[a \wedge b, b]$. Now consider the chains $\left\{a \wedge x_{n}\right\}$ in $(a)$ and $\left\{a \vee x_{n}\right\}$ in $[a, a \vee b]$. By the descending chain condition there is an $n$ such that for $m \geqq n$ we have $a \vee x_{n}=a \vee x_{m}$ and $a \wedge x_{n}=a \wedge x_{m}$. But $x_{m} \leqq x_{n}$ so that by modularity $x_{m}=x_{n}$ for $m \geqq n$. This shows that $a \vee b \in K$ and completes the proof. 
Proof of THe Theorem. Let $J$ denote the set of join irreducibles of $L$ and let $K$ be as in the Lemma. We intend to show that $J \subseteq K$. To show an $x \in J$ is an element of $K$ we may assume that each $y \in J, y<x$, is a member of $K$. This is because (ii) holds. Now by ( $i$ ) and the Lemma this implies that any $y<x$ is a member of $K$. It is now obvious that $x \in K$ since any chain $x \geqq x_{1} \geqq x_{2} \geqq \cdots$ is either constant or eventually in $K$. Hence $J \subseteq K$ and by (i) we have $K=L$. Therefore $L$ satisfies the descending chain condition.

Close examination of the proof shows that we can state:

COROLlary. If $L$ is any lattice in which (i) and (ii) hold and in which $K$ (as defined above) is an ideal, then L satisfies the descending chain condition.

We now give an example of a join-continuous lattice which satisfies $(i)$ and (ii) but not the descending chain condition. Let $L$ consist of the following elements in the real plane: (a) the origin $(0,0)$; (b) the line segment from $(0,1)$ to $(1,0)$ and; (c) the line segment from $(0,1)$ to $(1,2)$. It is easily checked that $L$ is a joincontinuous lattice with the order on $L$ induced by the pointwise order on the plane (a sketch is most helpful). The points $(t, 1-t), 0 \leqq t \leqq 1$, in the line segment described in (b) are atoms and thereby join-irreducible. An element $(t, 1+t)$, $0 \leqq t \leqq 1$, is given irredundantly as the join of $(0,1)$ and $(t, 1-t)$. It is now clear that (i) and (ii) hold in $L$, yet the line segment described in (c) has infinite descending chains so that the descending chain conditions does not hold.

\section{Reference}

[1] L. G. Kovács, 'The descending chain condition in join continuous modular lattices' $J$. Aust. Math. Soc. 10 (1969), 1-4.

Texas Tech University

Lubbock, Texas, 19409

U.S.A. 